\title{
CD133 is a temporary marker of cancer stem cells in small cell lung cancer, but not in non-small cell lung cancer
}

\author{
FEI CUI ${ }^{1}$, JIAN WANG ${ }^{1}$, DUAN CHEN ${ }^{2}$ and YI-JIANG CHEN ${ }^{1}$ \\ ${ }^{1}$ Department of Thoracic and Cardiovascular Surgery, the First Affiliated Hospital of Nanjing Medical \\ University, Nanjing, P.R. China; ${ }^{2}$ Department of Cancer Research and Molecular Medicine, \\ Norwegian University of Science and Technology, Trondheim, Norway
}

Received September 1,2010; Accepted November 2, 2010

DOI: $10.3892 /$ or.2010.1115

\begin{abstract}
Lung cancer is the most common cause of cancerrelated death worldwide. Current investigations in the field of cancer research have intensively focused on the 'cancer stem cell' or 'tumor-initiating cell'. While CD133 was initially considered as a stem cell marker only in the hematopoietic system and the nervous system, the membrane antigen also identifies tumorigenic cells in certain solid tumors. In this study, we investigated the human lung cancer cell lines A549, H157, H226, Calu-1, H292 and H446. The results of real-time PCR analysis after chemotherapy drug selection and the fluorescence-activated cell sorting analysis showed that CD133 only functioned as a marker in the small cell lung cancer line H446. The sorted $\mathrm{CD} 133^{+}$subset presented stem cell-like features, including self-renewal, differentiation, proliferation and tumorigenic capacity in subsequent assays. Furthermore, a proportion of the $\mathrm{CD} 133^{+}$cells had a tendency to remain stable, which may explain the controversies arising from previous studies. Therefore, the CD133+ subset should provide an enriched source of tumor-initiating cells among H446 cells. Moreover, the antigen could be used as an investigative marker of the tumorigenic process and an effective treatment for small cell lung cancer.
\end{abstract}

\section{Introduction}

Lung cancer incidence has increased sharply and the disease has become the most common cause of cancer-induced death worldwide (1). Lung cancer consists of two main pathological types. Small cell lung cancer (SCLC) accounts for 13-15\% of all lung cancers, but its clinical features tend to be more aggressive than the other type, non-small cell lung cancer (NSCLC) $(2,3)$. Recently, increasing evidence has rapidly accumulated all over the world showing a small subset of

Correspondence to: Professor Yi-Jiang Chen, The First Affiliated Hospital of Nanjing Medical University, No. 300, Guangzhou Avenue, Nanjing 210029, Jiangsu Province, P.R. China E-mail: yijiangchen2009@hotmail.com; cuidavil@hotmail.com

Key words: CD133, H446, cancer stem cells, tumorigenesis cells in the solid tumors. These so-called cancer stem cells can initiate the tumors with performing unique functions such as, self-renewal, asymmetric division, drug resistance and quiescence.

CD133, a highly conserved protein, has been proven and generally accepted as a marker for tumor stem cells in leukemia and glioblastoma $(4,5)$. This membrane antigen also contributes to the identification of the tumorigenic cells in some solid tumors, including prostate cancer, hepatocellular carcinoma and colon carcinoma (6-9).

With regard to lung cancer, both clinical analyses and laboratory studies have shown that CD133 plays a critical role in tumorigenesis in SCLC and NSCLC $(10,11)$. However, this theory is still controversial because CD133 is not a reliable tumor stem cell marker in lung cancer according to some studies, especially in NSCLC $(12,13)$. Therefore, we attempted to demonstrate the role of CD133 in SCLC and NSCLC and explore the possibility of sorting the tumorinitiating cells from lung cancer cells based on CD133 expression.

To exclude any synergistic effects (i.e., those effects resulting from angiogenesis), we worked with pure cell lines in this study. The tumor-initiating cells were initially enriched using chemotherapy agents, followed by analysis of CD133 mRNA expression in drug-selected cells (DSCs). We then performed a fluorescence-activated analysis to study the $\mathrm{CD}_{133^{+}}$subsets in all cell lines and selected the positive fractions. The specific cell lines with a $\mathrm{CD} 133^{+}$subset were sorted into a positive subset and a negative subset. With subsequent assays, we analyzed their respective biological and immune capacities. The study was designed to investigate the possibility of isolating tumor-initiating cells with the CD133 antibody and to provide evidence of the role of CD133 in lung cancer.

\section{Materials and methods}

Cell lines. Human lung cancer lines included in this study were: A549 (adenocarcinoma); H157 and H226 (squamous cell carcinoma); Calu-1 (epidermoid carcinoma); H292 (mucoepidermoid pulmonary carcinoma); and H446 (small cell lung cancer). Cells were obtained from the Shanghai Life Science Cell Bank of the Chinese Academy of Sciences and the American Type Culture Collection (ATCC). Cells were 

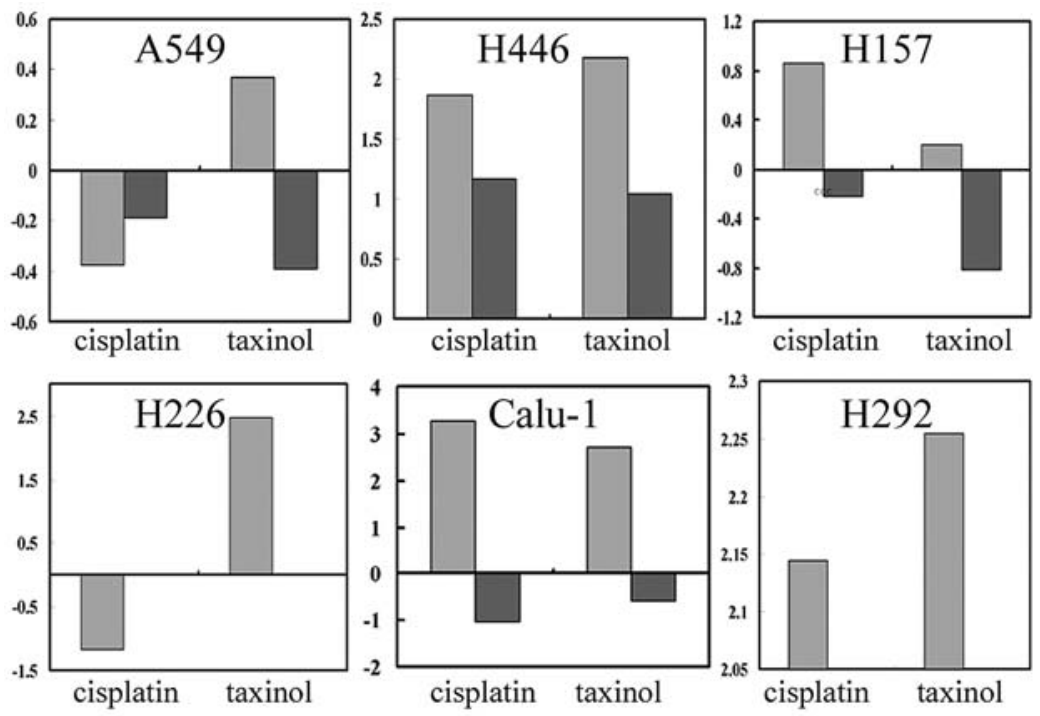

$\mathrm{ABCG} 2 \square \mathrm{CD} 133$

Figure 1. The mRNA expression of ABCG2 and CD133 in drug selected cells. The values were normalized with $18 \mathrm{~S}$ and converted to the common logarithm. ABCG2 mRNA expression up-regulated in all the respectively lines after treatment with cisplatin or taxinol, but only the CD133 mRNA expressed significantly higher in $\mathrm{H} 446$ cell line $(\mathrm{p}<0.05)$.

seeded in RPMI-1640 medium (Gibco), as recommended by the ATCC, that was supplemented with $1 \%$ penicillin/ streptomycin and 10\% fetal bovine serum (FBS, InvitrogenLife Technologies). All cells were incubated in a humidified incubator at $37^{\circ} \mathrm{C}$ in $5 \%$ carbon dioxide.

Chemotherapy drug selection. During the proliferative stage, cisplatin and taxinol were added at a final concentration of 1.25 and $2.5 \mu \mathrm{M}$, respectively (based on a preliminary experiment). After $72 \mathrm{~h}$, the living adherent cells (DSC, drugselected cells), $\sim 50 \%$ of the original number, were collected for the subsequent tests.

RNA extraction and real-time PCR analysis. We followed the standard real-time protocol to design the primers into product of $<200 \mathrm{bp}$. The sequences were referred to code AF027208 (AC133 antigen mRNA, CD133, GAAGAGCTTGCA CCAACAAA, AGATGACCGCAGGCTAGTTT) and NM_004827 (ATP-binding cassette sub-family G member 2 mRNA, ABCG2, GTTGTGATGGGCACTCTGAC, CCCT GTTAATCCGTTCGTTT) in gene bank.

Cells were harvested, and RNA extracted by the TRIzal (Invitrogen). Total RNA was treated with SuperScript III First-Strand Synthesis System on the iCycler PCR system (Bio-Rad) to process the synthesis of first strand followed by cDNA amplification according to the manual. Real-time PCR was operated on the iCycler iQ real-time PCR detection system (Bio-Rad) with SYBR supermix and Taq (Invitrogen) according to the manufacturer's instructions. The programmed thermal cycling conditions were $95^{\circ} \mathrm{C}$ for $30 \mathrm{sec}$ followed by 45 cycles of $5 \mathrm{sec}$ at $95^{\circ} \mathrm{C}, 34 \mathrm{sec}$ at $60^{\circ} \mathrm{C}$. Levels of expression were normalized to the 18S RNA (CGGCTACCA CATCCAAGGAA, GCTGGAATTACCGCGGCT).

Fluorescence-activated analysis and fluorescence-activated cell sorting (FACS). Parental cells were dissociated and suspended in phosphate-buffered saline (PBS) containing 2\% bovine serum albumin and adjusted to a final concentration of $10^{7}$ cells $/ \mathrm{ml}$. The specimens were labeled with anti-human CD133-PE antibody (eBioscience, USA) and incubated in the dark at $4^{\circ} \mathrm{C}$ for $30 \mathrm{~min}$. After washing twice with ice-cold PBS, the cells were sorted with the flow cytometer (BD FACS Calibur, USA). We only found a CD $133^{+}$subset in the $\mathrm{H} 446$ cell line (see Results), therefore the CD133+ and CD133subsets from the H446 cells were collected and cultured for the subsequent tumorigenesis assays. Cells were also stained with isotype control antibody (mIgG2b-PE, Caltag Laboratories) and analyzed by flow cytometry. We followed the same protocol described above to analyze the expression of ABCG2 in cell groups with purified mouse anti-human ABCG2 (BD Pharmingen $^{\mathrm{TM}}$, USA) and stained with PE-labeled goat antimouse IgG (BD Pharmingen).

\section{Tumorigenicity assay}

Growth curve. The sorted positive subset, negative subset and unsorted parental cells were cultured in 96-well plates, with $10^{3}$ cells per well. We assessed three wells from each group and performed the assay daily, and recorded the average data. The growth curve graphs were generated according to cell counts versus time.

Invasion assay. Cellular potential for invasion was determined for each subset of cells using 24-well plates and $8-\mu \mathrm{m}$ Matrigel invasion chambers (Corning). The chambers were coated with Matrigel, and the cells were dissociated and resuspended in RPMI-1640 medium containing $0.5 \%$ bovine serum albumin. Cells were seeded into upper chambers at $1 \times 10^{5}$ cells per chamber. Lower wells were filled with DMEM containing $10 \%$ FBS as a chemoattractive agent. Cells were incubated at $37^{\circ} \mathrm{C}$ with $5 \%$ carbon dioxide for $48 \mathrm{~h}$, and then non-invading cells were removed by swabbing the top layer of Matrigel. The membranes containing invading cells were fixed with methanol followed by staining with methyl violet. 

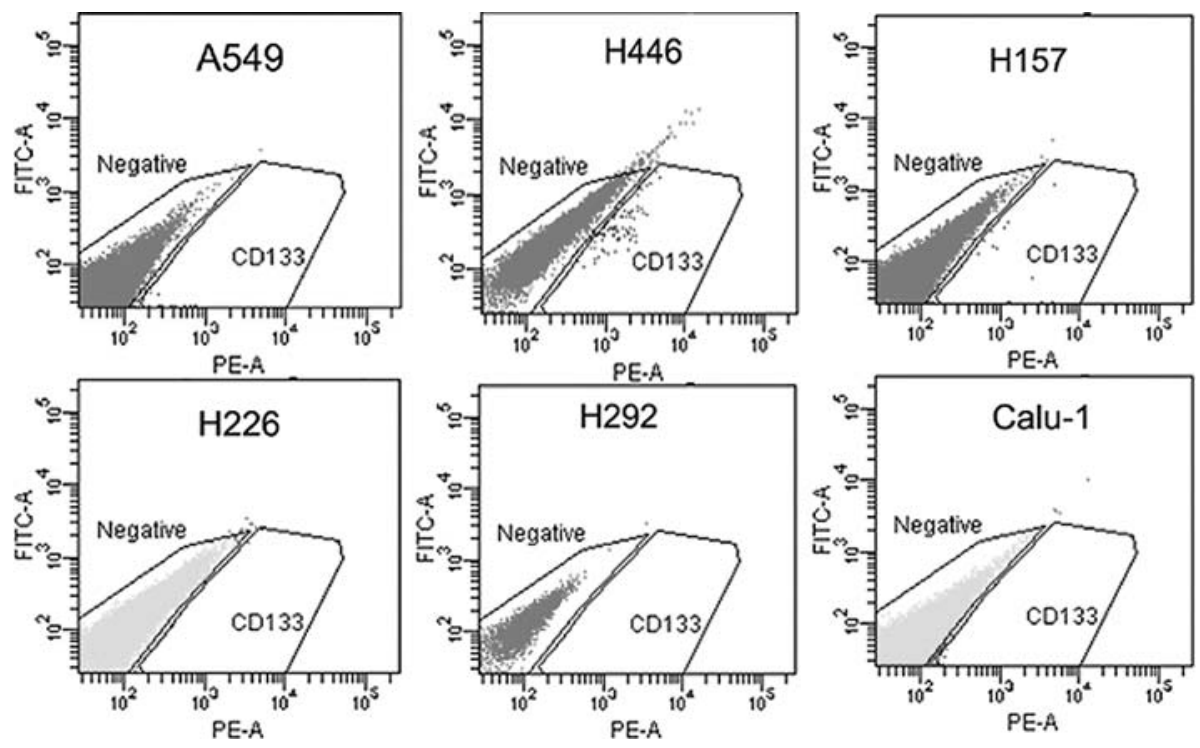

Figure 2. The CD133 analysis in the cell lines. The proportion was $0.4 \%$ in $\mathrm{H} 446$, and $<0.1 \%$ in the other 5 cell lines.

The membranes were then washed and mounted on slides. The invading cells were counted under a light microscope.

In vivo assay. We performed the in vivo experiment by following our institutional guidelines for the use of laboratory animals. The sorted positive subset, the negative subset and the parental cells were subcutaneously injected into male nude mice (CByJ.Cg-Foxn1 nu/J). The mice were supplied by the National Resource Center for Mutant Mice in Nanjing University of China at three weeks of age. The mice were inoculated with $1 \times 10^{3}, 5 \times 10^{3}, 1 \times 10^{4}, 5 \times 10^{4}, 1 \times 10^{5}$ and $5 \times 10^{5}$ cells, respectively, and three mice were inoculated per group. When the xenograft tumors grew to $8 \mathrm{~mm}$, or after 6 weeks of inoculation, the tumor was resected under intraperitoneal anesthesia. The tumor tissues were embedded in paraffin and sectioned, followed by H\&E staining. The final slides were viewed with a microscope. The positive subset cells were also analyzed with the CD133 antibody by fluorescence cytometry.

Statistical analysis. Data are presented as the mean \pm SD. The data were analyzed with SPSS 17.0 software. T-test and ANOVA were used to compare differences between groups. $\mathrm{p}<0.05$ was considered statistically significant.

\section{Results}

Increased mRNA expression in the drug-selected cells. The CD133 mRNA (Prominin-I) was expressed at very low levels in the cell lines. Nevertheless, the level of CD133 mRNA varied after treatment with cisplatin and taxinol. Only H446 cells showed a significant increase in CD133 mRNA expression after treatment. However, the ABCG2 mRNA was expressed at much higher levels in all six treated cell lines compared to untreated cells (Fig. 1).

Isolation and differential characterization of the $\mathrm{CD} 133^{+} \mathrm{H} 446$ cells. We analyzed CD133 protein expression in various cell lines by flow cytometry and found that the positive rate was $0.883 \pm 0.491 \%$ in H446 cells (Figs. 2 and 5), but the positive subset had nearly disappeared by the 15th generation (more than one month). No positive fraction was found in the other five cell lines (Fig. 2). At the same time, ABCG2 expression, in the H446 $\mathrm{CD} 133^{+}$subset, was much higher than the parental cells. The positive and negative subsets were sorted by labeling with the anti-human CD133 antibody and cultured in 96-well plates under identical conditions to compare their repopulation capacity. According to the above results in H446 cells, the DSCs of this line were analyzed as well. This assay demonstrated a similar level of CD133 expression and a higher level of ABCG2 expression in these DSCs as the parental H446 cells.

We also re-analyzed both subsets within two weeks. The positive proportion of the sorted positive subset of the cells had decreased from 100 to $18 \%$ after 7 days with a similar reduction in ABCG2 expression. The proportion declined to $2.1 \%$ by the end of day 14 (Fig. 5). In contrast, the positive proportion of the negative-sorted subset had risen from zero to $1.0 \%$ after 14 days. Since the $\mathrm{CD} 133^{+}$cells regenerated both $\mathrm{CD} 133$ - cells and $\mathrm{CD} 133^{+}$cells, the result indicated that the $\mathrm{CD} 133^{+}$cells had renewal and differentiation capacities.

The $\mathrm{CD} 133^{+}$subset has a greater capacity for proliferation than the CD133- subset. In the same medium of a 96-well plate, the respective proliferative ability of the two subsets and the parental cells were investigated via growth curve analysis (Fig. 6B). Based on the cell counts versus time graph, the parental cells initially multiplied faster than both subsets. After several population doublings, the parental cells and both subsets entered an exponential growth period. As expected, the $\mathrm{CD}_{133^{+}}$subset showed greater proliferative ability than the negative subset $(p=0.011)$ However, the number of positive subset cells only slightly exceeded the parental cells after the 10th day of seeding, without a statistically significant difference $(\mathrm{p}=0.129)$. Our observation period lasted two weeks, and the multiplication rate (shown as the percent of the slope in the graph) began to decline after the 12th day due to contact inhibition. 


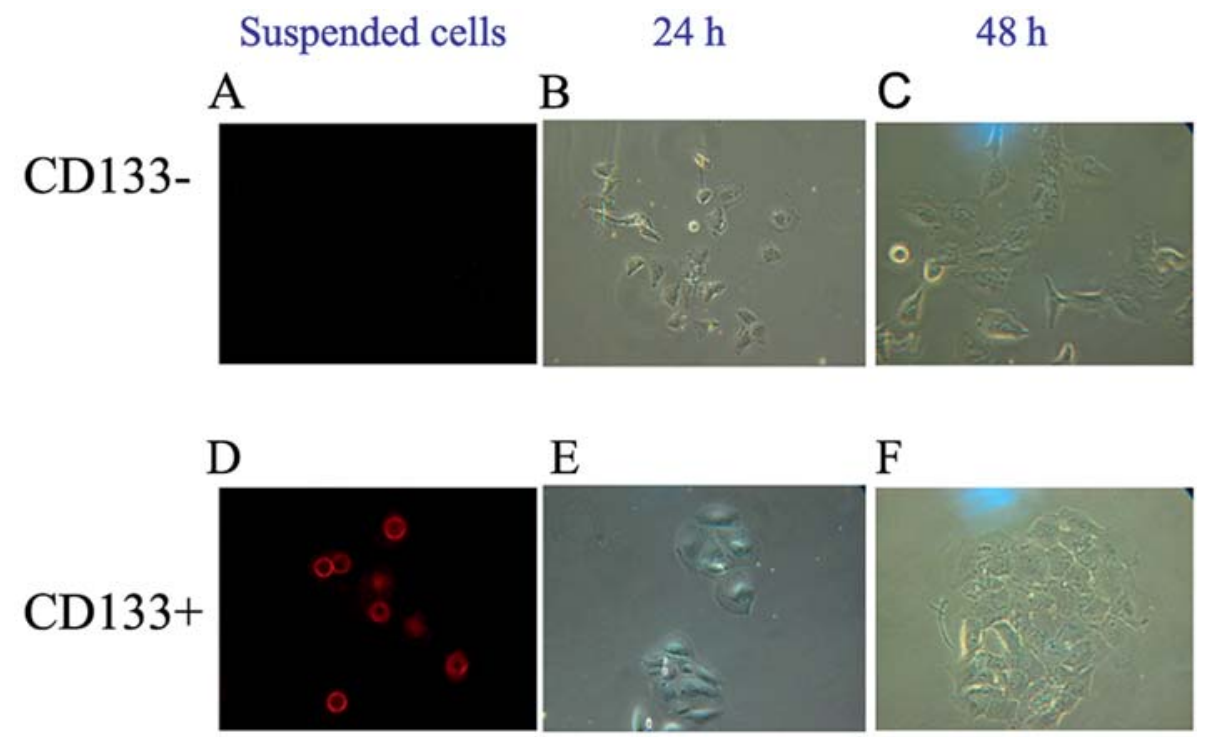

Figure 3. Photographs of sorted CD133+ and CD133- cells in H446. The images of suspended CD133- (A) and CD133+ (D) cells in H446 were acquired by a fluorescence microscope. There were more streak impurities (arrows) and fragments attaching the cells in CD133+ cell cultures (E and F) than in CD133cultures (B and C). Later, the impurities were washed off by PBS when the medium was changed. The negative cell clones proliferated much faster than the the positive after sorting for $24-48 \mathrm{~h}$.

A

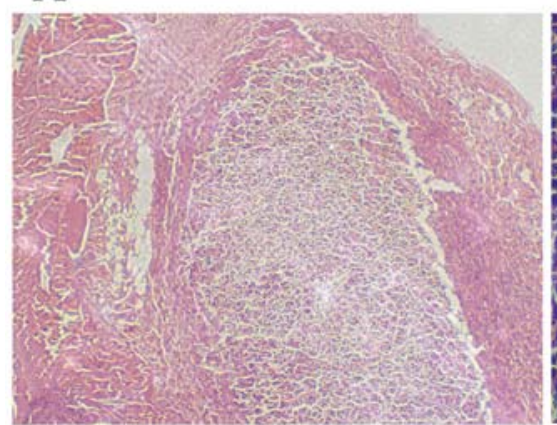

$\mathrm{C}$

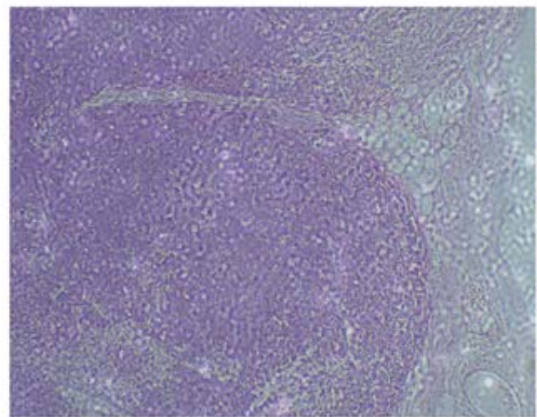

\section{$\mathrm{E}$}

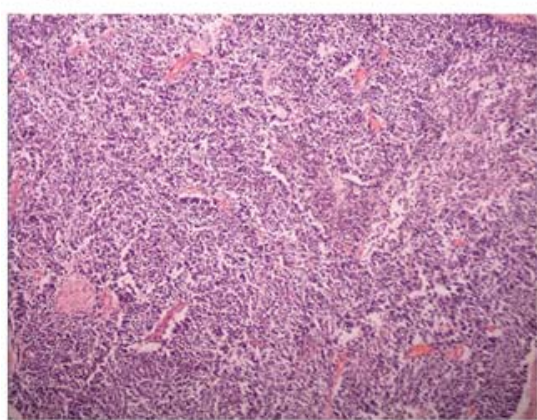

\section{B}

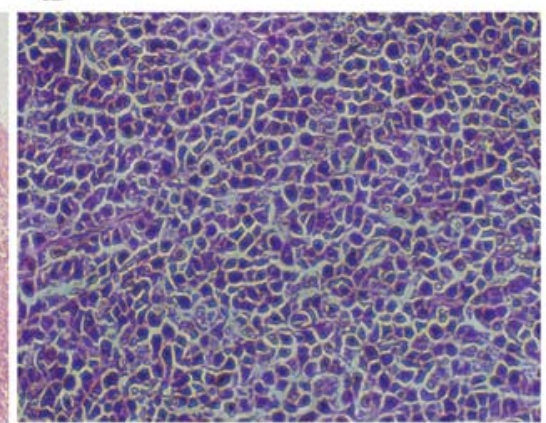

$\mathrm{D}$

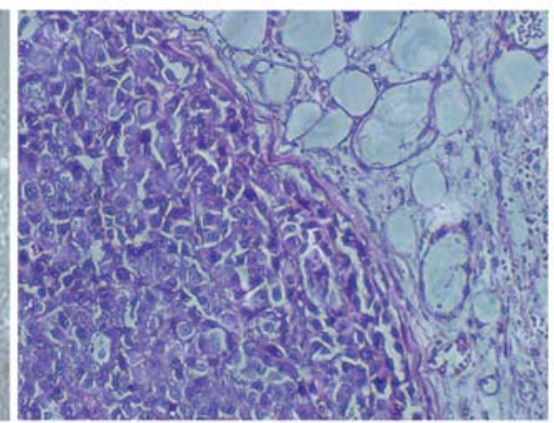

$\mathrm{F}$

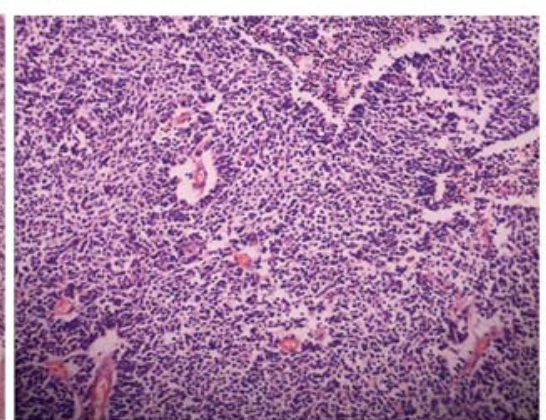

Figure 4. Histopathology of nude mouse xenografts and human small cell lung cancer. Both CD133- (A and B) and CD133+ (C and D) nude mouse tumor lost the appearance of typical human small cell lung cancer ( $\mathrm{E}$ and $\mathrm{F})$ and displayed the characteristics of undifferentiated tumor cells. 
A

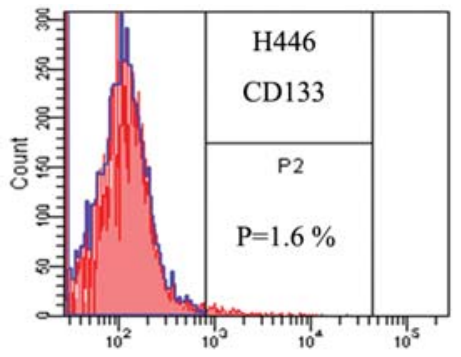

D

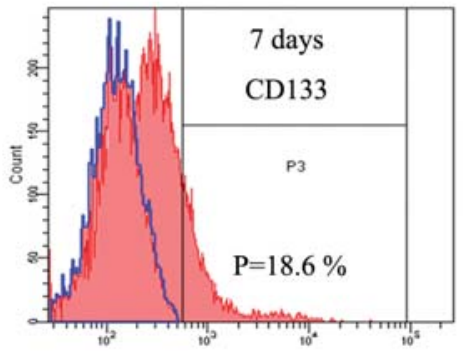

G

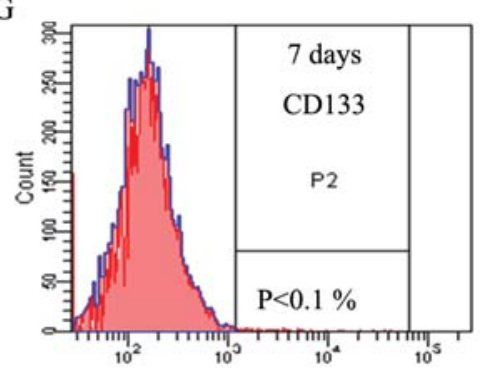

B
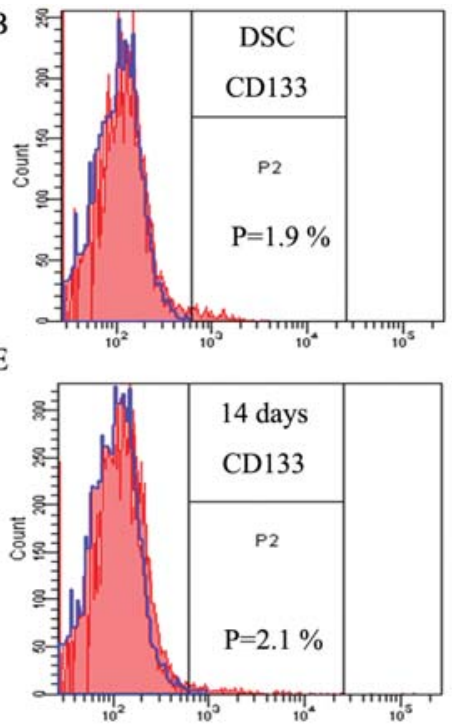

$\mathrm{H}$

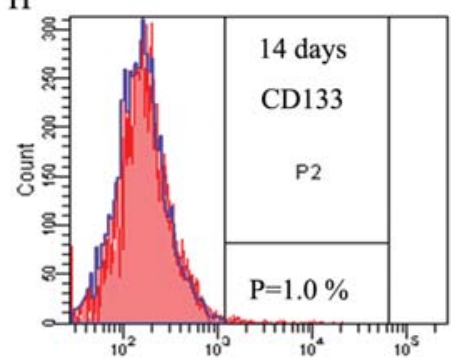

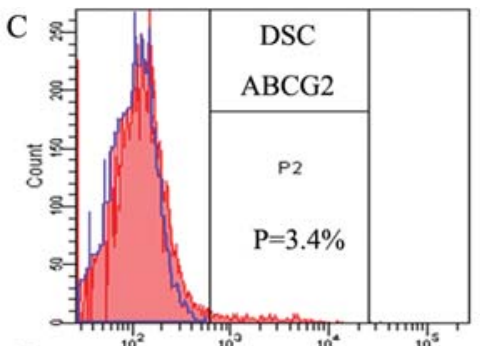

F

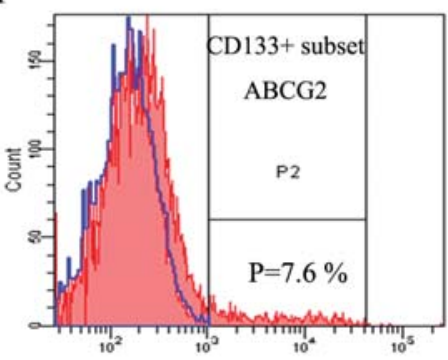

I

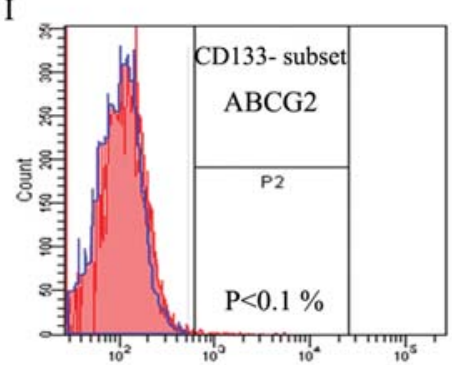

Figure 5. The variation of CD133 and ABCG2 expression in H446 (the outline of control was drawn in blue line to exclude the control result). The CD133+ proportion of $\mathrm{H} 446$ was $0.883 \pm 0.491 \%$ (A), and the $\mathrm{CD} 133^{+}$proportion of $\mathrm{H} 446$ drug selected cells (DSC) was $0.933 \pm 0.565 \%$ (B), which equaled to the parental cells $(\mathrm{p}=0.504 ;>0.05)$. The expression of ABCG2 in H446 DSCs: the proportion of ABCG2 expression was $3.4 \%$ (C). The CD133 expression in sorted positive subset did not remain stable: the proportion had dropped to $18.6 \%$. After 14 days, it was only $2.1 \%$ (D and E). On the contrary, the proportion rose from 0.1 to $1.0 \%$ in the $\mathrm{CD} 133^{-}$subset after 14 days (G and $\mathrm{H}$ ). The ABCG2 expression in both sorted subsets after 14 days: the proportion of sorted $\mathrm{CD}_{133^{+}}$subset was $7.6 \%$, and the proportion of the CD133- subset was $<0.1 \%$ (F and I).

Increased invasive ability and greater in vivo tumorigenic potential of $\mathrm{CD} 133^{+}$cells. In the invasion assay, we counted the number of cells within the membrane. We found that over 3 times more $\mathrm{CD} 133^{+}$cells $(\mathrm{n}=1258 \pm 582)$ had traversed the Matrigel than CD133 cells ( $\mathrm{n}=380 \pm 200)$, and the positive cells were $\sim 1.2$ times more than the parental cells $(n=1045 \pm 377)$. There was a statistically significant difference between the positive subset and the negative subset $(\mathrm{p}<0.01)$, but there was no significance between the positive and parental cells $(p=0.331)$. Therefore, the positive fraction showed more active invasive ability than the negative fraction (Fig. 6A).

The tumorigenic potential of $\mathrm{CD} 133^{+}$cells was so distinct compared with the other groups that tumors could form using only $5 \times 10^{3}$ of these cells $(2 / 3$ mice $)$ in immune-deficient nude mice within 3 weeks of hypodermic injection (Fig. 6C). Even so, it is surprising that the hypodermal tumor never expanded $>2 \mathrm{~mm}$ after having emerged. We did not plant $>10^{5}$ positive cells for two reasons: 1) The culture time was too long to obtain that number without a conclusive higher CD133 expression (proven by the CD133 analysis in the 7th day and 14th day), and 2) Since all these cells are malignant, $10^{5}$ cells are generally enough to form a tumor, that is why injecting $>10^{5}$ cells had no significance in comparison to tumorigenesis between tumor cells. The assay showed that the CD133- subset had less tumor-initiating capacity (Table I) because this subset could only form a tumor when $10^{5}$ cells were injected (1/3 mice). This number is at least 20 -fold more than the positive cells. We resected the xenograft tumor derived from the positive subset and reanalyzed CD133 expression. We found that the positive proportion had returned to normal levels (Fig. 6D), which was equal to the level seen in the parental H446 cells. The histopathology of the xenografts is shown in Fig. 4. Although there was no difference between the positive tumors and negative tumors, the tumor cells were obviously discernable from the normal human SCLC cells under a magnification of x200.

\section{Discussion}

In the present study, we only found a $\mathrm{CD} 133^{+}$subset in the H446 SCLC cell line. The CD133+ subset possessed a greater tumorigenic potential than the CD133- subset in the in vivo assay. The positive fraction could differentiate into positive and negative fractions as asymmetric division. Furthermore, compared with the negative subset, the positive subset displayed a more aggressively invasive and proliferative ability, as shown with the proliferation assays. The above results demonstrated that the tumor stem cells could be enriched in the $\mathrm{CD}_{133}{ }^{+}$cells, in accordance with the current standards to cancer stem cells (14). 


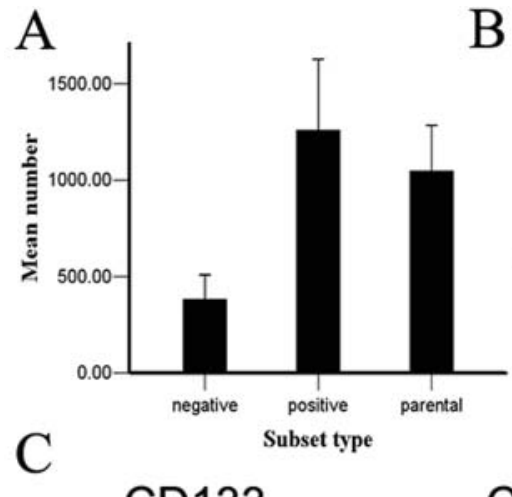

CD133-

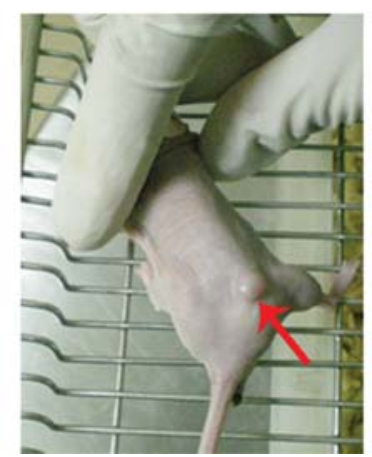

B

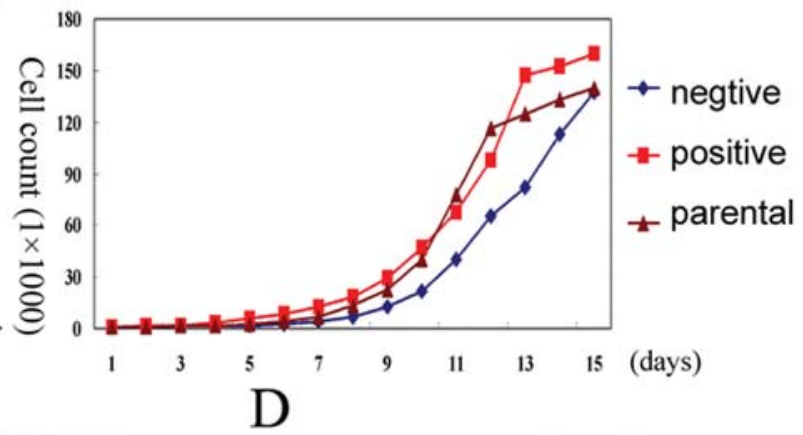

CD133+

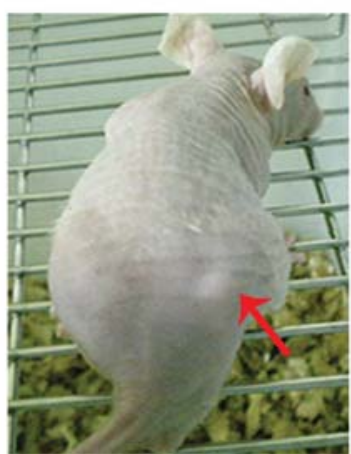

Xenograft cells
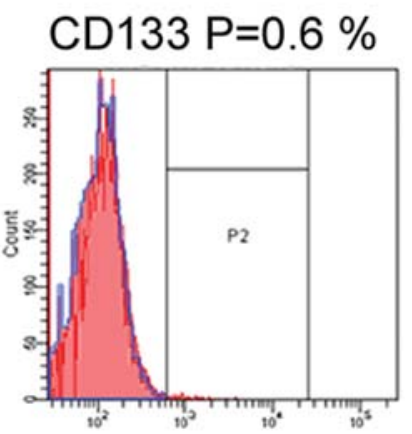

Figure 6. Tumorigenic features of the in vitro and in vivo assays. In the invasion assay, the data (A) showed CD133+ subset traversed more than both $\mathrm{CD} 133^{-}$ subset $(\mathrm{p}<0.01)$, therefore this subset had more active invasive ability. However, there was no difference between positive subset and parental cells ( $\mathrm{p}=0.33$; $>0.05$ ). (B) The growth curve graph, the parental cells multiplied faster than both subsets at first because of the cell injury of sorting process. Then the $\mathrm{CD} 133^{+}$subset proliferated significantly faster than the CD133- subset during the whole observation period ( $<0.05$ ). Even the CD133 $3^{+}$subset had exceeded the parental cells, but the slope began to decline after 12 days. Groups came to plateau after the population after $1.4 \times 10^{5}$. (C) Just $5 \times 10^{3} \mathrm{CD} 133^{+}$cells could grow to a tumor (arrow) in the nude mouse, however, it needed $10^{5}$ parental cells to form a tumor compared with the former. The CD133 expression of nude xenograft tumor cells was $0.6 \%(\mathrm{D})$.

Table I. The result of in vivo assay.

\begin{tabular}{lcccccc}
\hline & \multicolumn{5}{c}{ Cell number for injection (tumor diameter, mm) } \\
\cline { 2 - 7 } & $10^{3}$ & $5 \times 10^{3}$ & $10^{4}$ & $5 \times 10^{4}$ & $10^{5}$ & $5 \times 10^{5}$ \\
\hline CD133 $^{+}$ & $2 \mathrm{~mm}(2 / 3)$ & $2 \mathrm{~mm}(3 / 3)$ & $2 \mathrm{~mm}(3 / 3)$ & $\mathrm{N} / \mathrm{A}^{\mathrm{a}}$ & $\mathrm{N} / \mathrm{A}$ \\
Parent & - & - & - & - & $6 \mathrm{~mm}(3 / 3)$ & $8 \mathrm{~mm}(3 / 3)$ \\
CD133 & - & - & - & - & $4 \mathrm{~mm}(1 / 3)$ & $6 \mathrm{~mm}(3 / 3)$ \\
\hline
\end{tabular}

${ }^{\mathrm{a}}$ Explained in Results.

In contrast, we did not find a CD133+ subset in NSCLC cell lines such as A549, H157, H226, H292 and Calu-1. Therefore, CD133 should not be considered a reliable tumor stem cell marker in NSCLC.

The lung cancer stem cell hypothesis is controversial. Some believe that these so-called stem cells come from bone marrow stem cells or embryonic stem cells (15-17), while others insist that they may derive from bronchoalveolar progenitor cells (BASCs) (18). SCLC and NSCLC, the two subtypes of lung cancer, are completely different from one another in terms of tumor biological behavior, histological appearance and treatment. SCLC may be more like a systemic disease that affects the respiratory system rather than a respiratory disease, especially in the advanced stages. All these results suggest that the sources of progenitor cells in SCLC and NSCLC are not the same, necessitating two separate methods for isolating the tumor-initiating cells, but this hypothesis has not been confirmed. There is also some evidence that NSCLC may be related to BASCs but not to CD133+ cells $(13,18)$. The abnormal expression of CD133 in clinical NSCLC patients may not be due to an independent effect but rather to some synergetic effects.

It has not been confirmed whether the cancer stem cells in SCLC come from bone marrow stem cells or embryonic stem cells. CD133 was initially identified as a stem cell marker in the hematopoietic system. It is also found among immature 
cells in some human organs, such as the bone marrow and spleen (19). The role of CD133 in leukemia is undoubtedly important. Since some chemotherapy protocols are similar between leukemia and SCLC, understanding the role of CD133 in SCLC may be valuable. Hayashi et al reported on the existence of a CD133+ ${ }^{+}$subset in two SCLC lines, NCIH82 and NCI-H69, with higher tumorigenicity than the CD133- subset (20). Also, according to our present results, the $\mathrm{CD} 133^{+}$subset only was found in the SCLC cell line. We found only the CD133 mRNA up-regulation after the treatment with cisplatin and taxinol in the H446 SCLC line, whereas the ABCG2 mRNA was overexpressed in all drugselected lines.

ABCG2 is one of the confirmed markers in a wide variety of stem cells (21), and several studies have shown that the co-action of ABCG2 and CD133 may induce tumor chemoresistance $(22,23)$. The drug selection process enriches stem-like cells in tumors (24). Theoretically, although the cancer stem cell hypothesis comes from the definition of human stem cells, clinicians are interested in it because some particular fraction of cells can survive treatment with cytotoxic agents. The existence of cancer stem cells explains why chemotherapy cannot completely eliminate solid tumors. Thus, chemoresistance should be one of the features of the so-called cancer stem cells. Some studies have suggested that this specific fraction possesses this function for two reasons: first, that there are some membrane transport proteins, such as ABCG2, that can transport the chemotherapeutic agents out of the cell, and secondly due to quiescence (discussed below). Thus, according to our results, the co-increase in CD133 mRNA and ABCG2 mRNA in H446 drug-selected cells implies that CD133 may play a role in the enriched tumor-initiating cells, but not in other cell lines (A549, H157, H226, H229 and Calu-1). Although we cannot yet explain why normal CD133 protein expression is maintained in the drug-selected H446 cells (Fig. 5B), this result may be logically considered as a sign of the induction or enrichment of immature cells.

We also found some additional interesting phenomena related to so-called quiescence. Although the correlation between CD133 and quiescence has not been confirmed, Dooley and colleagues have demonstrated that CD34 expression increases as CD34- cells shift from quiescence to proliferation (27). Since CD133 cells are regarded as a subset of the $\mathrm{CD} 34^{+}$cells, the role of CD133 in quiescence is likely to attract attention. Based on our results, some interesting phenomena were found, which might be related to quiescence.

A small number of positive cells were present in the sorted negative subsets after several generations, and their proportion rose to the level of the positive ratio of the parental cell population. This phenomenon suggested that the potential $\mathrm{CD} 133^{+}$cells may be latent in the negative fraction or that quiescent CD133 cells may be revived after microenvironmental changes. On the contrary, the proportion of CD133 cells in the sorted positive subset was reduced to normal level at the same time, which indicated that the positive ratio tended to remain stable. The positive cells decrease in proportion due to the asymmetrical division, which means that one cancer stem cell can divide to produce one cancer stem cell and one mature tumor cell (14). Therefore, their reduction makes the cells generally appear quiescent.

The xenograft tumors generated from the $\mathrm{CD} 133^{+}$subset could not expand infinitely in the in vivo assay. As few as thousands of cells were enough to form a tumor up to $2 \mathrm{~mm}$ in diameter within 3 weeks. However, the xenograft tumor could not expand beyond this size, whereas the unsorted parental cells could form a tumor up to $6 \mathrm{~mm}$ in diameter upon the injection of $10^{5}$ cells. We can also interpret this phenomenon as a general down-regulation of the CD133+ subset because the positive rate of CD133 expression in the $\mathrm{CD}_{133^{+}}$nude mouse xenograft tumors was $0.6 \%$ (Fig. 6D). This result indicated that the proportion of CD133-positive cells had decreased significantly within six weeks, even in an in vivo microenvironment.

The above results suggest that CD133 may be a temporary cancer stem cell marker that is not always expressed throughout the tumor proliferation process. Even in normal organs, CD133 is rapidly down-regulated and disappears completely during the differentiation and maturation period (25). Hence, it is typically quiescent, which may contribute to drug resistance (26). When the quiescence is broken by certain factors, the CD133 mRNA is up-regulated, and the protein will be expressed, after which it will play a role in some unknown signaling pathway. If the microenvironment later reverses to a more stable condition, the active tumor-initiating cells will again become dormant and down-regulate CD133 mRNA. Our results can be illustrated with this hypothesis: The status of quiescence makes $\mathrm{CD} 133^{+}$cells more like stem cells than the negative one. Based on the above points, CD133 may play a role in the tumor initiation process rather than the tumor maintenance process. Thus, we refer to its cancer stem cell marker status as temporary.

Some other controversies involving CD133 can also be resolved if the role of CD133 in quiescence can be confirmed. For example, Shmelkov et al found that both $\mathrm{CD} 133^{+}$and CD133- metastatic tumor subpopulations can form colonospheres in in vitro cultures and were capable of long-term tumorigenesis in a NOD/SCID serial xenotransplantation model. Moreover, metastatic CD133- cells form more aggressive tumors than the positive cells and express typical phenotypic markers of cancer-initiating cells (28). We consider all of these results to be reliable and reasonable as long as the point mentioned above is accepted. In vivo and in vitro assays take at least two weeks, which is long enough to stimulate the up-regulation of CD133 after quiescence or to down-regulate it from the active situation. We believe these dynamics explain why there is almost no difference between the positive and negative xenografts in terms of pathologic tumor morphology. From our study, we can see that the variations in CD133 expression tend towards stability not only in the positive subset but also in the negative subset. The malignant cells are likely to lose some stem cell-like features when the proportion of CD133+ cells decreases. Thus, in our assay, the tumors generated from $\mathrm{CD} 133^{+}$cells could not grow any larger after three weeks. This area of study will require further investigation to determine the causes of this phenomenon.

In accordance with the opinion that CD133 is a temporary marker, any future targeted therapies directed against CD133 
should be considered for the maintenance of the quiescent status of the malignant cells (particularly including the latent $\mathrm{CD}_{133^{+}}$cells) instead of merely eliminating certain cells. This treatment approach may transform cancer into a chronic disease, much like hypertension and diabetes.

\section{Acknowledgements}

We appreciate Hai-Wei Yang PhD, Zhen-Hua Tian MD and Jie Chen MD for technical assistance on flow cytometry, and Dr Jie Yao for help with CD133 antibody. We thank Cun-yu Wang PhD (Laboratory of Molecular Signaling, Division of Oral Biology and Medicine, University of California, Los Angeles, CA, USA) for editing assistance and helpful comments.

\section{References}

1. Boyle P and Levin B: World Cancer Report 2008. Vol. 390 , International Agency for Research on Cancer, Lyon, 2008.

2. Rosti G, Bevilacqua G, Bidoli P, Portalone L, Santo A and Genestreti G: Small cell lung cancer. Ann Oncol 17 (Suppl 2): ii5-ii10, 2006.

3. Simon GR and Wagner H: Small cell lung cancer. Chest 123: S259-S271, 2003.

4. Lapidot T, Sirard C, Vormoor J, et al: A cell initiating human acute myeloid leukaemia after transplantation into SCID mice. Nature 367: 645-648, 1994

5. Beier D, Hau P, Proescholdt M, et al: CD133+ and CD133 glioblastoma-derived cancer stem cells show differential growth characteristics and molecular profiles. Cancer Res 67: 4010-4015, 2007.

6. Yin S, Li J, Hu C, et al: CD133 positive hepatocellular carcinoma cells possess high capacity for tumorigenicity. Int J Cancer 120: 1436-1440, 2007.

7. Ricci-Vitiani L, Lombardi DG, Pilozzi E, et al: Identification and expansion of human colon-cancer-initiating cells. Nature 445: 111-115, 2007.

8. Collins AT, Berry PA, Hyde C, Stower MJ and Maitl NJ: Prospective identification of tumorigenic prostate cancer stem cells. Cancer Res 65: 10946-10951, 2005.

9. Suetsugu A, Nagaki M, Aoki H, Motohashi T, Kunisada T and Moriwaki H: Characterization of $\mathrm{CD} 133^{+}$hepatocellular carcinoma cells as cancer stem/progenitor cells. Biochem Biophys Res Commun 351: 820-824, 2006.

10. Dome B, Timar J, Dobos J, et al: Identification and clinical significance of circulating endothelial progenitor cells in human non-small cell lung cancer. Cancer Res 66: 7341-7347, 2006.

11. Hohenberger P, Nowak K, Rafat N, et al: The level of circulating endothelial progenitor cells is increased in human lung cancer patients and correlates with the progression of the disease. J Clin Oncol 26: 22055, 2008.
12. Meng X, Wang X and Wang Y: More than $45 \%$ of A549 and H446 cells are cancer initiating cells: Evidence from cloning and tumorigenic analyses. Oncol Rep 21: 995-1000, 2009.

13. Qiang-gang D, Ming Y, Qin G, Jin Z and Ming-xia Y: Isolation and identification of human lung adenocarcinoma stem cells. Tumor 28: 1-7, 2008 (In Chinese).

14. Bhattacharyya S and Khanduja KL: New hope in the horizon: cancer stems cells. Acta Biochim Biophys Sin (Shanghai) 42: 237-242, 2010.

15. Paguirigan A, Beebe DJ, Liu B and Alexander C: Mammary stem and progenitor cells: tumour precursors? Eur J Cancer 42: 1225-1226, 2006

16. Mackenzie IC: Stem cell properties and epithelial malignancies. Eur J Cancer 42: 1204-1212, 2006.

17. Haura E: Is repetitive wounding and bone marrow-derived stem cell mediated-repair an etiology of lung cancer development and dissemination? Med Hypotheses 67: 951-956, 2006.

18. Kim CF, Jackson EL, Woolfenden AE, et al: Identification of bronchioalveolar stem cells in normal lung and lung cancer. Cell 121: 823-835, 2005.

19. Miraglia S, Godfrey W, Yin AH, et al: A novel fivetransmembrane hematopoietic stem cell antigen: isolation, characterization, and molecular cloning. Blood 90: 5013-5021, 1997.

20. Hayashi T, Tao H, Jida M, et al: Expression of CD133, a possible marker for cancer stem cells (CSCs), in small cell lung cancer (SCLC) cell lines and non-small cell lung cancer (NSCLC) cell lines. J Clin Oncol 27: e22100, 2009.

21. Zhou S, Schuetz JD, Bunting KD, et al: The ABC transporter $\mathrm{Bcrp1} / \mathrm{ABCG} 2$ is expressed in a wide variety of stem cells and is a molecular determinant of the side-population phenotype. Nat Med 7: 1028-1034, 2001.

22. Frank NY, Margaryan A, Huang Y, et al: ABCB5-mediated doxorubicin transport and chemoresistance human malignant melanoma. Cancer Res 65: 4320-4333, 2005.

23. Doyle LA and Ross DD: Multidrug resistance mediated by the breast cancer resistance protein BCRP (ABCG2). Oncogene 22: 7340-7358, 2003.

24. Levina V, Marrangoni AM, DeMarco R, Gorelik E and Lokshin AE: Drug-selected human lung cancer stem cells: cytokine network, tumorigenic and metastatic properties. PLoS One 3: e3077, 2008.

25. Mizrak D, Brittan M and Alison MR: CD133: molecule of the moment. J Pathol 214: 3-9, 2008.

26. Dean M, Fojo T and Bates S: Tumour stem cells and drug resistance. Nat Rev Cancer 5: 275-284, 2005.

27. Dooley DC, Oppenlander BK and Xiao M: Analysis of primitive CD34- and $\mathrm{CD}^{-} 4^{+}$hematopoietic cells from adults: gain and loss of CD34 antigen by undifferentiated cells are closely linked to proliferative status in culture. Stem Cells 22: 556-569, 2004.

28. Shmelkov SV, Butler JM, Hooper AT, et al: CD133 expression is not restricted to stem cells, and both $\mathrm{CD}_{133^{+}}$and $\mathrm{CD} 133$ metastatic colon cancer cells initiate tumors. J Clin Invest 118 : 2111-2120, 2008 